INPLASY

PROTOCOL

To cite: Zhang et al.

Comparison of magnesium status between athletes and untrained population: $A$ systematic review and metaanalysis. Inplasy protocol 202140055. doi:

10.37766/inplasy2021.4.0055

Received: 08 April 2021

Published: 09 April 2021

Corresponding author:

Haixin Zhang

1711516022@sus.edu.cn

Author Affiliation:

Shanghai University of Sport

Support: NSFC (31671242, 31971097).

Review Stage at time of this submission: Piloting of the study selection process.

Conflicts of interest:

None declared.

\section{Comparison of magnesium status between athletes and untrained population: A systematic review and metaanalysis}

Zhang, HX1; Wang, RW2; Guo, SS3; Tian, QQ4; Liu, TM5; Wang, R6.

Review question / Objective: To determine the magnesium status of athletes compared with untrained population, we conducted this meta-analysis.

Condition being studied: When the metabolism accelerates during exercise, magnesium in the human body is redistributed to adapt the needs of accelerated metabolism, and magnesium requirement may increase due to additional magnesium losses through sweat and urine. However, conflicting results have been reported in dietary magnesium intake and magnesium concentrations of athletes compared with untrained population. As far as athletes are concerned, a suboptimal or even deficient magnesium status may lead to inefficient energy metabolism and affect athletic ability. This study is the first to perform a systematic review and metaanalysis on magnesium status of athletes compared with untrained population.

INPLASY registration number: This protocol was registered with the International Platform of Registered Systematic Review and Meta-Analysis Protocols (INPLASY) on 09 April 2021 and was last updated on 09 April 2021 (registration number INPLASY202140055).

\section{INTRODUCTION}

Review question / Objective: To determine the magnesium status of athletes compared with untrained population, we conducted this meta-analysis.

Condition being studied: When the metabolism accelerates during exercise, magnesium in the human body is redistributed to adapt the needs of accelerated metabolism, and magnesium requirement may increase due to additional magnesium losses through sweat and urine. However, conflicting results have been reported in dietary magnesium intake and magnesium concentrations of athletes compared with untrained population. As far 
as athletes are concerned, a suboptimal or even deficient magnesium status may lead to inefficient energy metabolism and affect athletic ability. This study is the first to perform a systematic review and metaanalysis on magnesium status of athletes compared with untrained population.

\section{METHODS}

Search strategy: Relevant studies were identified by electronically searching the following data sources: PubMed (From inception to 5 April 2021) ; Web of Science (From inception to 5 April 2021); SPORTDiscus (From inception to 5 April 2021); Cochrane Library (From inception to 5 April 2021). The search terms involved "magnesium", "athlet*", "exercis*", "train*", "physical activity", "sport*". Searches were restricted to human participants and the English language.

Participant or population: Participants are healthy adults. In addition, participants in study group are male and/or female athletes, and participants in control group are untrained adults.

Intervention: Athletes take part in regular exercising training, while untrained population don't taking part in exercising training.

Comparator: Magnesium status of athletes was compared with that of untrained population.

Study designs to be included: Observational (such as cross-sectional) will be included.

Eligibility criteria: Studies were included if they met the following criteria: (1) published in English; (2) participants are healthy adults; (3) participants in study group are athletes and participants in control group are untrained adults; (4) outcomes: at least one relevant magnesium outcome; (5) participants can't take supplements containing magnesium or other minerals ; (6) study design: observational (such as cross-sectional) study including study group and control group.

Information sources: Relevant studies were searched from the databases of PubMed, Web of Science, SPORTDiscus and Cochrane Library from inception to 5 April 2021.

Main outcome(s): Dietary magnesium intake, serum/plasma magnesium concentration, erythrocyte magnesium concentration, 24-hour urinary magnesium excretion.

Additional outcome(s): None.

Data management: We used a standardized form for extracting data of the included articles. The following data were extracted: the study authors, year of publication, region of study, characteristics of participants ( sample size, sex, age), measured outcomes (dietary magnesium intake, serum/plasma magnesium concentration, erythrocyte magnesium concentration and 24-hr urinary magnesium excretion).

Quality assessment / Risk of bias analysis: The risk of bias in individual studies will be assessed by two authors independently according to the GRADE approach, The grades of risk included low risk, unclear risk and high risk.

Strategy of data synthesis: Continuous outcomes will be analysed by calculating the mean difference (MD) when the same instrument is used to measure outcomes or the standardized mean difference (SMD) when different instruments are used to measure the outcomes. The uncertainty will be expressed with $95 \%$ confidence intervals $(95 \% \mathrm{Cl})$. The outcome measures from the individual trials will be combined through meta-analysis where possible using a fixed-effect model unless there is significant statistical heterogeneity, in which case a random-effects model will be used. A P value of the $x^{2}$ test less than 0.05 indicates a significant statistical heterogeneity. If a meta-analysis is not possible, the results from clinically 
comparable trials will be described qualitatively in the text.

Subgroup analysis: Stratified analyses will be completed for subgroups of types of athletes.

Sensitivity analysis: When there are sufficient studies, we will carry out sensitivity analysis to test the robustness of studies according to the quality of method, the sample size and the selection of missing data. And the fluctuation of results will be observed.

Country(ies) involved: China.

Keywords: Athletes; Untrained population; Magnesium status.

Contributions of each author:

Author 1 - Haixin Zhang.

Email: 1711516022@sus.edu.cn

Author 2 - Ruwen Wang.

Email: 2011516014@sus.edu.cn

Author 3 - Shanshan Guo.

Email: shanshan_guo@fudan.edu.cn

Author 4 - Qianqian Tian.

Email: 1811516019@sus.edu.cn

Author 5 - Tiemin Liu.

Email: tiemin_liu@fudan.edu.cn

Author 6 - Ru Wang.

Email: wangru@sus.edu.cn 